\title{
EVALUACIÓN DE UNA INTERVENCIÓN BASADA EN LA PSICOLOGÍA POSITIVA, PARA LA DISMINUCIÓN DE CONDUCTAS ANTISOCIALES EN ADOLESCENTES
}

\author{
Gerardo Riffo Allende \\ Universidad Pedro de Valdivia, Chile \\ gerardo.riffo@upv.cl
}

Recepción Artículo: 23 abril 2021

Admisión Evaluación: 23 abril 2021

Informe Evaluador 1: 25 abril 2021

Informe Evaluador 2: 26 abril 2021

Aprobación Publicación: 27 abril 2021

\section{RESUMEN}

En este estudio, el autor tiene como objetivo evaluar la efectividad de una intervención basada en la psicología positiva dirigida a disminuir la frecuencia de conductas antisociales en adolescentes. El diseño utilizado para evaluar esta intervención es el de línea base múltiple a través de 3 diadas de adolescentes y el análisis realizado se hace en términos de significación clínica y estadística. Los resultados permiten concluir que los cambios observados se deben a la intervención basada en la psicología positiva, ya que los 6 adolescentes disminuyeron sus niveles de conducta antisocial subtipo agresión, robo y violación de normas y además existe evidencia de un aumento en los niveles de felicidad y optimismo. Esto sugiere que este tipo de intervención puede ser prometedora para adolescentes con estas conductas. Se discuten las implicaciones y limitaciones del estudio.

Palabras claves: conducta antisocial; psicología positiva; adolescentes; felicidad; optimismo

\section{ABSTRACT}

Evaluation of an intervention based on positive psychology, for reducing antisocial behavior in adolescents. In this study, the author aims to evaluate the effectiveness of an intervention based on positive psychology aimed at reducing the frequency of antisocial behaviors in adolescents. The design used to evaluate this intervention is that of multiple baseline across 3 dyads of adolescents and the analysis performed is done in terms of clinical and statistical significance. The results allow us to conclude that the changes observed are due to the intervention based on positive psychology, since the 6 adolescents decreased their levels of antisocial behavior, subtype aggression, theft and violation of norms, and there is also evidence of an increase in happiness levels. and optimism. This suggests that this type of intervention may be promising for adolescents with these behaviors. The implications and limitations of the study are discussed.

Keywords: antisocial behavior, positive psychology, adolescents, happiness, optimism 


\section{INTRODUCCIÓN}

Las conductas antisociales en adolescentes constituyen un problema psicológico, social y cultural de gran interés en el ámbito científico. Existe en los últimos años un aumento en la magnitud de este fenómeno 0 , al menos, una elevada sensibilización hacia el ejercicio de estas conductas, las cuales son potenciadas por los procesos del ciclo evolutivo en la cual se desarrollan (Andreu et al., 2010; Nasaescu et al., 2020).

Rodríguez et al. (2012) refieren que los adolescentes que presentan comportamientos antisociales en edades tempranas y por tiempo prolongado, ingresan a ser parte de un grupo en alto riesgo para continuar con las mismas conductas y de mayor gravedad durante la edad adulta. Este mismo grupo también, presentaría alto riesgo para otros problemas, como dificultades académicas, dificultades familiares, consumo de drogas y alcohol, además de comportamientos sexuales peligrosos (Blatný et al, 2012; Chavarín-García \& Gálvez-Hernández, 2018; Díaz García \& de la Villa Moral-Jiménez, 2018; Estévez y Emler, 2011; Gázquez et al, 2016; Hasking, Scheier y Abdallah, 2011; Morán et al., 2016; Piotrowska et al., 2015; Ribas et al., 2015)

Asimismo, Martínez (2010) y Servicio Nacional de Menores (2019) refieren que en los últimos años se produce un significativo cambio, principalmente por los problemas comportamentales que se dan en niños y adolescentes en centros residenciales de protección para este grupo etario, y actualmente es un tema que suscita gran preocupación en el colectivo de profesionales que trabajan en esta área.

Garaigordobil (2017) y Rodríguez et al. (2016) señalan que en las residencias de protección se ha detectado una alta incidencia de conductas de rechazo y aislamiento, así como las referentes a comportamientos y estados ansiosos o depresivos. Las conductas que tienen una mayor frecuencia en esta muestra son las que tienen que ver con comportamientos de carácter agresivo. Destaca además, la presencia de problemas de atención e interacción social.

En estos centros se brinda especial énfasis en analizar los problemas de conducta de los niños, niñas y adolescentes en el sistema de residencias de protección, lo cual es de importancia por la relación demostrada con el ajuste psicosocial, por las posibles consecuencias sobre el éxito o el fracaso de la propia medida de protección, sobre las relaciones diarias con las personas con las que conviven los acogidos, por la trascendencia de la evaluación para la detección temprana de dificultades de adaptación y finalmente para la toma de decisiones en el proceso de intervención psicosocial (Servicio Nacional de Menores, 2010; Servicio Nacional de Menores, 2019).

Los datos aportados por diferentes países de la Unión Europea (Fondo de las Naciones Unidas para la Infancia, 2011) señalan el interés por analizar el incremento y la gravedad de los problemas psicológicos de los niños, niñas y adolescentes protegidos y tutelados por la administración pública, con el fin de diseñar programas de intervención adecuados a sus necesidades y a la de su sistema familiar.

El Servicio Nacional de Menores (2010) señala que los niños, niñas y adolescentes en centros de protección presentan una mayor incidencia y gravedad de problemas de conducta que el resto de la población de su misma edad, lo que indica la necesidad de potenciar recursos específicos y especializados, orientados a las necesidades de intervención en esta población. Los últimos estudios señalan que el $53 \%$ de los usuarios de estos centros han sido víctimas de maltrato físico o psicológico grave, algunos de ellos incluso con causal de ingreso de abuso sexual o situación de abandono.

Además, destaca que presentan deserción escolar, provienen de familias disfuncionales y tienen escasas redes de apoyo, limitando su desarrollo y sus propias capacidades. Es por esto que las intervenciones que se realicen a los y las adolescentes, deben estar enfocados a su bienestar y al 
fortalecimiento de sus habilidades, recursos y potencialidades (López \& Rodríguez-Arias; 2010; Martínez, 2010).

Es por este antecedente que urge la necesidad de desarrollar métodos de promoción del bienestar a través del entrenamiento o focalización de emociones positivas. Asimismo, de realizar propuestas sistematizadas de intervenciones para que las personas logren ser resistentes a la adversidad y puedan prevenir la aparición presente o futura de emociones negativas, particularmente, en la población adolescente que presenta conductas antisociales. (Giménez et al., 2010)

Carrea y Mandil (2011) señalan que, si bien la psicología positiva es un paradigma general orientado a refocalizar la perspectiva de la investigación, la prevención y la práctica clínica en los recursos y fortalezas generales, existen una serie de modelos terapéuticos que se sustentan explícitamente en sus planteamientos (Toribio et al., 2018). Todos ellos apuntan al reforzamiento de los recursos y experiencias orientadas al bienestar y la promoción de emociones positivas. Asimismo, los datos de la investigación en psicología positiva sugieren que, al favorecer el desarrollo de un adolescente en un contexto de bienestar, se disminuyen las posibilidades de contraer psicopatología y se promueve el desarrollo de sus potencialidades (Bohlmeijer et al., 2017; Garaigordobil \& Maganto, 2016; Salanova y Llorens, 2016).

En el presente estudio, se realiza una intervención basada en la psicología positiva a adolescentes que presentan conductas de tipo antisocial. Se evalúa el efecto de la intervención en la disminución de estas conductas. También se examina si los niveles de felicidad y optimismo aumentan posterior a la intervención.

\section{MÉTODO}

\section{Participantes}

Participaron 3 diadas de adolescentes con diagnóstico clínico de conducta antisocial realizado por profesionales de un centro de salud pública con experiencia en este tipo de conductas. Los 6 adolescentes eran usuarios de centros residenciales de protección a la infancia perteneciente a la red del Servicio Nacional de Menores de la Región Metropolitana de Chile.

La muestra estuvo compuesta por 2 diadas de sexo masculino y 1 diada de sexo femenino. Sus edades comprendían entre 12 y 16 años, todos ellos oriundos de Santiago. Respecto a su ocupación, los 6 participantes se encontraban estudiando al momento de la investigación. $\quad \mathrm{P}$ a $\mathrm{r}$ a efectos del presente estudio también se dividió a los integrantes en dos grupos, cada uno de ellos quedó conformado por un número de tres adolescentes (2 hombres y 1 mujer).

Se utilizaron seis criterios de experto para el ingreso de cada diada a la presente investigación: que estén ingresados a un mismo centro residencial de protección; sean personas del mismo sexo; presenten menos de seis meses de diferencia en su edad cronológica; presenten igual nivel educacional; presenten menos de seis meses de diferencia en su ingreso al centro de protección; y presenten similar causal de ingreso al centro residencial de protección. 
Tabla 1.

Características de los participantes

\begin{tabular}{|c|c|c|c|c|c|}
\hline $\begin{array}{l}\text { Datos } \\
\text { Diada }\end{array}$ & Genero & $\begin{array}{c}\text { Edad } \\
\text { cronológica }\end{array}$ & Nivel educacional & $\begin{array}{c}\text { Fecha de } \\
\text { ingreso }\end{array}$ & Causal de ingreso \\
\hline A1 & Masculino & $\begin{array}{c}16 \text { años } \\
10 \text { meses }\end{array}$ & $\begin{array}{c}2^{\circ} \text { año de } \\
\text { enseñanza media }\end{array}$ & Abril 2016 & $\begin{array}{l}\text { Negligencia, } \\
\text { maltrato físico }\end{array}$ \\
\hline $\mathrm{A} 2$ & Masculino & $\begin{array}{l}16 \text { años } \\
6 \text { meses }\end{array}$ & $\begin{array}{c}2^{\circ} \text { año de } \\
\text { enseñanza media }\end{array}$ & $\begin{array}{l}\text { Agosto } \\
2016\end{array}$ & $\begin{array}{l}\text { Negligencia, } \\
\text { maltrato físico }\end{array}$ \\
\hline B1 & Fem & $\begin{array}{l}14 \text { años } \\
2 \text { meses }\end{array}$ & $\begin{array}{c}8^{\circ} \text { año de } \\
\text { enseñanza básica }\end{array}$ & Junio 2018 & $\begin{array}{l}\text { Abuso sexual, } \\
\text { maltrato físico }\end{array}$ \\
\hline B2 & Femenino & $\begin{array}{l}14 \text { años } \\
0 \text { meses }\end{array}$ & $\begin{array}{c}8^{\circ} \text { año de } \\
\text { enseñanza básica }\end{array}$ & $\begin{array}{l}\text { Marzo } \\
2018\end{array}$ & $\begin{array}{l}\text { Abuso Sexual, } \\
\text { maltrato físico }\end{array}$ \\
\hline $\mathrm{C} 1$ & Masculino & $\begin{array}{l}13 \text { años } \\
7 \text { meses }\end{array}$ & $\begin{array}{r}7^{\circ} \mathrm{a} \\
\text { enseñan }\end{array}$ & $\begin{array}{l}\text { Septiembre } \\
2017\end{array}$ & $\begin{array}{l}\text { VIF, maltrato } \\
\text { físico }\end{array}$ \\
\hline $\mathrm{C} 2$ & Masculino & $\begin{array}{l}13 \text { años } \\
4 \text { meses }\end{array}$ & $\begin{array}{c}7^{\circ} \text { año de } \\
\text { enseñanza básica }\end{array}$ & Junio 2017 & $\begin{array}{l}\text { VIF, maltrato } \\
\text { físico }\end{array}$ \\
\hline
\end{tabular}

\section{Configuración y materiales}

Se solicitó permiso a la Dirección Metropolitana del Servicio Nacional de Menores para obtener autorización para la realización del presente estudio. Se ejecutó una revisión de datos socio-demográficos de los adolescentes con la finalidad de reunir los criterios de selección de la muestra para contactar a las personas pre-seleccionadas e invitarlas a participar del estudio. Se proporciona a los adolescentes la información éticamente pertinente.

Todas las sesiones se llevaron a cabo en el centro residencial de protección de cada diada de adolescentes, específicamente se utiliza la sala de entretenimiento o de juegos, esto en función de la conveniencia de los funcionarios del centro como también para la observación de todo el proceso de intervención. Se selecciona el horario de 17:00 a 18:00 horas, horario donde todos los adolescentes del centro se encuentran en espacio de entretención o esparcimiento.

El transcurso de las sesiones de evaluación de línea base, tratamiento y seguimiento fue registrada por tres observadores, los cuales eran licenciados en psicología. Estos 3 observadores fueron entrenados y capacitados durante 6 semanas previas al inicio de la fase de línea base. Para asegurar la fiabilidad de las observaciones, los tres evaluadores no conocieron los tiempos de cada fase de intervención ni de la asignación de los adolescentes a los grupos.

\section{Instrumentos}

Escala de felicidad subjetiva. Es una medida de carácter global de felicidad subjetiva, considerando la definición de felicidad desde la perspectiva de quien responde. Consta de 4 ítems con respuesta tipo Likert, donde su corrección se realiza mediante la sumatoria de los puntajes obtenidos de cada respuesta y estos se dividen en el número total de ítems (Lyubomirsky \& Lepper, 1999). En cuanto a las propiedades psicométricas, el estudio preliminar realizado por Vera-Villarroel, CelisAtenas y Córdova-Rubio (2011) refiere un alpha de Cronbach de 0.71 para adultos y de 0.87 para adolescentes, como también una estructura factorial pura, lo que indica adecuación de esta escala para su utilización en la población chilena.

Test de orientación vital revisado. (LOT-R) (Scheier, Carver \& Bridges, 1994) para medir la predisposición generalizada hacia las expectativas de resultados positivos, en la versión española de Otero et al. (1998). Consta de 6 ítems (más 4 ítems de relleno) que proporciona una puntuación de optimismo disposicional en escala Likert de 5 puntos, desde 0 (muy en desacuerdo) hasta 4 (muy 
de acuerdo) (Chico, 2002). Las propiedades psicométricas de este test en población chilena muestran un alpha de Cronbach de 0.65 y respecto al análisis factorial, surge una estructura bifactorial asociada al optimismo y al pesimismo, por lo que presenta adecuación para su uso en nuestro país (Vera-Villarroel, Córdova-Rubio \& Celis-Atenas, 2009).

Escala de registro de conductas antisociales. Consta de 21 ítems divididos en 3 subescalas de 7 ítems cada una. Cada una de ellas evalúa un tipo de transgresión: Agresión, las que puede realizar a sus pares y/o adultos; Robo - Destrucción de la propiedad; y violación de normas. En esta escala se marca la presencia de la conducta objetivo cuando esta se presenta, a lo menos una vez, dentro del intervalo de tiempo correspondiente. Para interpretar los resultados se suman los puntajes de los reactivos correspondientes a cada subescala, estos puntajes van de 0 a 12 (a mayor puntaje mayor frecuencia de conductas antisociales).

\section{Procedimiento}

El presente estudio empleo un diseño de línea base múltiple, de tipo entre sujetos, en la cual, una conducta meta se selecciona para dos o más sujetos (o grupos) en un mismo contexto. Una vez que se logra un estado estable de respuesta bajo condiciones de línea base, la variable independiente se aplica a uno de ellos, mientras que las condiciones de línea base permanecen para los otros sujetos. (Kazdin, 2001). Se utilizó esta técnica para observar y evaluar los cambios en las conductas antisociales de los adolescentes. Las fases de intervención consideradas en este diseño fueron:

Línea base

Esta fase se realizó previa a la intervención en psicología positiva, en la cual se registro la frecuencia de las conductas antisociales como también, de los niveles de felicidad y optimismo de los adolescentes mediante la aplicación de los instrumentos descritos anteriormente. Las sesiones de la línea base se llevaron a cabo durante 31 días (un mes) para los participantes del grupo I y de 37 días (un mes y una semana) para los participantes del grupo II, en las dependencias del centro residencial de protección.

Tratamiento

Esta etapa correspondió a la implementación de la intervención en psicología positiva con los adolescentes previamente seleccionados, la cual se define como un procedimiento que aborda específicamente la conducta antisocial en un grupo de adolescentes, mediante el uso de estrategias y herramientas que incorporan intervenciones enfocadas en las emociones positivas, compromiso, relaciones interpersonales, sentido y la propia realización (Seligman, 2008).

Se estructuró en forma de psicoterapia de tipo individual de 5 sesiones, con una duración de 50 minutos cada una y una periodicidad de dos veces a la semana. La intervención estará a cargo de un psicólogo, el cual guiará los contenidos y actividades realizados en ésta. Las sesiones fueron de carácter semiestructurado, con el fin de poder adaptarlas a las necesidades de los adolescentes y de los procesos estipulados en el centro residencial de protección.

Seguimiento

Posterior a la finalización de la intervención en psicología positiva, se realizó una medición diaria de las conductas antisociales de 14 días (dos semanas) para los participantes del grupo I y de 07 días (una semana) para los participantes del grupo II.

Se realizó una segunda fase de seguimiento a los 15, 30, 45 y 60 días finalizada la fase de tratamiento, con el fin de evaluar la frecuencia de las conductas antisociales como también, de los niveles de felicidad y optimismo de los participantes, mediante los instrumentos ya nombrados.

\section{Confiabilidad y validez}

Para evaluar la significación clínica de los cambios generados por la intervención en psicología 
positiva se llevaron los datos obtenidos a un gráfico de línea base múltiple. Por tratarse de un diseño de línea base múltiple, no se registró el mismo número de sesiones de línea base y seguimiento para todos los participantes (generando datos perdidos en las sesiones de dichas fases), por lo cual se reemplazaron los datos perdidos por la media obtenida por cada uno de estos sujetos en cada una de las fases.

Para asegurar la fiabilidad de las observaciones, se utilizó el método de observadores independientes durante las 3 fases de evaluación. Los observadores independientes alcanzaron un 93\% de acuerdo, lo que es adecuado según los criterios de Forehand \& Mc Mahon (1981).

Finalmente, para evaluar la significación estadística de los cambios obtenidos por la intervención se realizaron análisis con la prueba de Análisis de Varianza (ANOVA) para medidas repetidas mediante el programa estadístico SPSS versión 22.0 para Windows. Esta prueba estadística permitió estudiar el efecto de uno o más factores cuando al menos uno de ellos es un factor intra-sujetos.

\section{RESULTADOS}

Conductas antisociales. Durante el inicio del estudio, los tres adolescentes del grupo I presentaban una alta frecuencia de conductas antisociales tipo agresión con un porcentaje promedio de un $50,6 \%$ del tiempo, en el subtipo robo-destrucción de propiedad alcanzo un valor de $34,2 \%$ del tiempo y en el subtipo violación de normas este fue de un 51,1\% del tiempo (rango entre 4 y 9 conductas). Tras el inicio de la fase de intervención, la frecuencia de las conductas antisociales de los adolescentes fue disminuyendo gradualmente hasta llegar a niveles mínimos (5 puntos 0 menos en todos los casos). Al finalizar el tratamiento, el promedio de la frecuencia de las conductas del subtipo agresión y del subtipo robo-destrucción de propiedad era de un 32,5\% del tiempo, y en el subtipo violación de normas este alcanzo un 29,6\% del tiempo. En la fase de seguimiento, las conductas se mantuvieron en similares condiciones a la de tratamiento, (5 puntos 0 menos en todos los casos) incluso luego de 30 días finalizado dicho proceso.

Los tres adolescentes del grupo II presentaban una alta frecuencia de los tres subtipos de conductas antisociales medidas en línea base: agresión con un porcentaje promedio de un 52,9\% del tiempo, robo-destrucción de propiedad alcanzo un valor de 34,9\% del tiempo y en violación de normas este fue de un $52,5 \%$ del tiempo. Iniciado el tratamiento, las conductas antisociales fueron disminuyendo gradualmente hasta niveles mínimos (5 puntos o menos en todos los casos) y al concluir la intervención, la frecuencia promedio de las conductas del subtipo agresión y del subtipo robo-destrucción de propiedad era de un 33,3\% del tiempo, el subtipo robo-destrucción de propiedad fue de 29,6\% del tiempo, y en el subtipo violación de normas este alcanzo un 30,7\% del tiempo. Estos resultados se mantuvieron durante las mediciones de seguimiento realizadas a los 15 y 30 días después de finalizada la intervención.

60 días después de finalizado el tratamiento, se realizó en todos los participantes del grupo I y II una nueva medición de los tres subtipos de conductas antisociales, con el propósito de detectar cuales permanecían en el repertorio conductual de los adolescentes. Los resultados manifestaron que las conductas antisociales de tipo agresión y robo - destrucción de propiedad se mantenían en parámetros similares a fase de intervención con una frecuencia promedio de un $25 \%$ (rango entre 2 y 3 conductas) y en el tipo violación de normas se visualiza una frecuencia de $22 \%$ (rango entre 2 y 4 conductas).

Felicidad y optimismo. En la fase de línea base los seis adolescentes presentaban bajos índices de felicidad ( 2 de un máximo de 7 ) y optimismo (3 de un máximo de 12). Tras el inicio de la fase de intervención, la frecuencia fue aumentando gradualmente hasta llegar a niveles moderados en felicidad (4 puntos) y de optimismo (5 puntos). El promedio de la frecuencia de estos índices se 
mantienen hasta finalizar el tratamiento e incluso en algunos adolescentes, estos continuaron en aumento. Estos resultados se mantuvieron durante la fase de seguimiento realizado a los 15 días y al mes después de finalizada la intervención (4 y 5 puntos respectivamente). A los 60 días de haber finalizado el tratamiento, se realiza en todos los participantes del grupo I y II una nueva medición de felicidad y optimismo, con la finalidad de visualizar se permanecían los niveles obtenidos durante el tratamiento. Los resultados manifestaron que se mantenían en parámetros similares.

Aceptabilidad del tratamiento. EI ANOVA de un factor con medidas repetidas confirmo diferencias significativas en todas las categorías examinadas. En la conducta antisocial subtipo agresión respecto al nivel de los efectos del tratamiento intra-sujetos y evaluando las condiciones línea base y tratamiento tenemos un nivel de significación de $p=0,0000$, el tamaño del efecto observado es mayor a 0,8 y la potencia de prueba es de 1,00. En la conducta antisocial subtipo violación de normas respecto al nivel de los efectos del tratamiento intra-sujetos y evaluando las condiciones línea base y tratamiento tenemos una con un nivel de significación de $p=0,0000$, el tamaño del efecto observado es mayor a 0,8 y la potencia de prueba es de 1,00. En la conducta antisocial subtipo robodestrucción de propiedad respecto al nivel de los efectos del tratamiento intra-sujetos y evaluando las condiciones línea base y tratamiento tenemos un nivel de significación de $p=0,0000$, el tamaño del efecto observado es mayor a 0,8 y la potencia de prueba es de 1,00. Por lo tanto, los resultados aseveran que existe una disminución de dichas conductas en los 6 adolescentes.

Respecto a los niveles de felicidad y optimismo, se observan diferencias estadísticamente significativas entre las condiciones de línea base y tratamiento a nivel de grupos con un nivel de significación de $p=0,0000$, tamaño del efecto observado mayor a 0,8 y la potencia de prueba de 1,00. Por lo tanto, los datos confirman la existencia de un aumento de dichos índices en los 6 adolescentes.

Gráfico 1.

Promedio de cantidad de conductas antisociales subtipo agresión exhibidas por los adolescentes en las diferentes fases de la intervención.

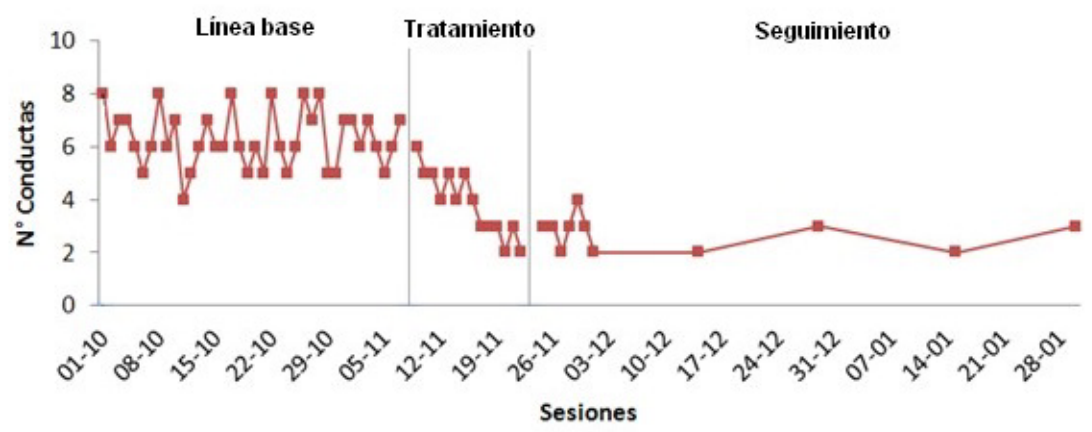


Gráfico 2.

Promedio de cantidad de conductas antisociales subtipo robo - destrucción de propiedad exhibidas por los adolescentes en las diferentes fases de la intervención.

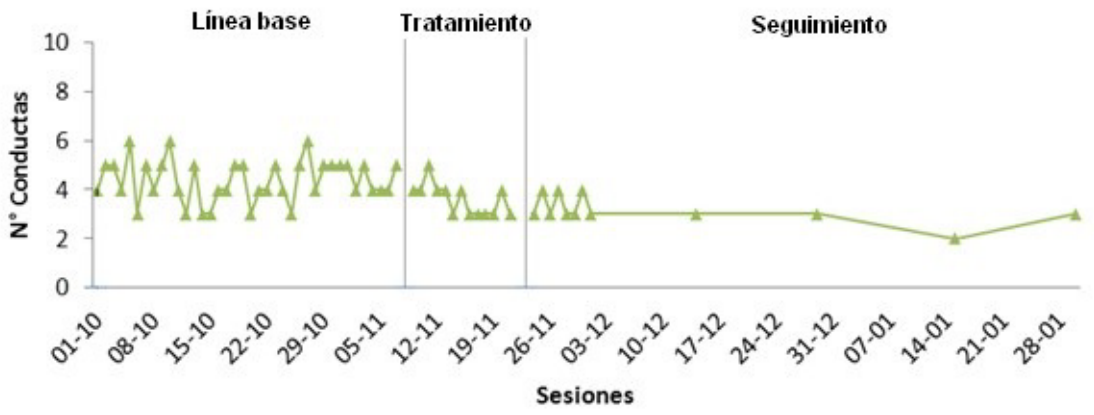

Gráfico 3.

Promedio de la cantidad de conductas antisociales subtipo violación de normas exhibidas por los adolescentes en las diferentes fases de la intervención.

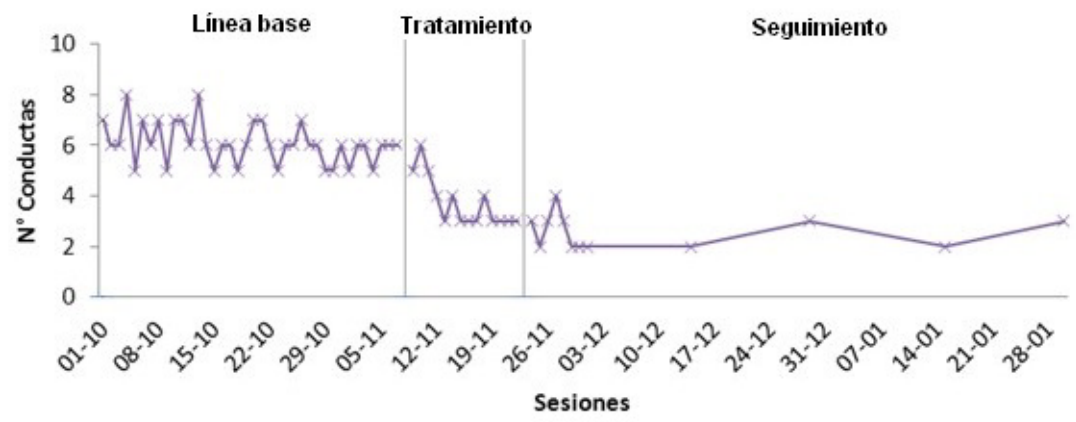

\section{DISCUSIÓN}

Esta investigación documenta que la disminución de las conductas antisociales de los adolescentes es producto de la intervención basada en la psicología positiva, lo cual es significativo en términos tanto de observación como a nivel estadístico. La evaluación de los datos de la intervención se ha realizado en términos de significación estadística y clínica. Los diferentes indicadores utilizados (cualitativos y cuantitativos) en esta investigación nos permiten avalar que los programas basados en la evidencia reportan no sólo resultados estadísticamente significativos, sino que también un alto valor en cuanto a su importancia práctica (significación clínica). Esto confirma los planteamientos de Fredrickson (2001), quien señala que al favorecer el desarrollo de un adolescente en un contexto de bienestar, se disminuyen las posibilidades de contraer psicopatología y se promueve el desarrollo de sus potencialidades.

Respecto a las variables felicidad y optimismo, se observó un aumento en sus niveles desde la primera medida de tratamiento hasta la última medida de seguimiento en ambos grupos de adolescentes. Lo anteriormente expuesto, presenta similitud con los resultados obtenidos de los estudios internacionales realizados por Diener y Seligman, 2002; Donaldson et al., 2015; Kahneman et al., 2006; Lyubomirsky et al., 2005; Rashid, 2015; Seligman et al., 2005; Seligman et al., 2006; Sheldon 
et al., 2002, los cuales plantean que las intervenciones basadas en la psicología positiva resultan altamente eficaces en el aumento de dichas variables.

Esta investigación se constituye en un primer acercamiento en Chile en el ámbito de las conductas antisociales desde la psicología positiva, la cual aporta una herramienta útil y concreta que, en corto tiempo y a bajo costo, da respuesta a este tipo de patología, este antecedentes es importante, ya que, puede ser más fácil para los profesionales de centros de salud implementar este tipo de intervención.

\section{LIMITACIONES}

Este estudio tenía un número de limitaciones. En primer lugar, el diseño línea base múltiple presenta problemas respecto a la validez externa, por lo que dificulta generalizar los resultados a otros sujetos o población. La evaluación de la conducta antisocial en el presente estudio presenta altos costos debido a las capacitaciones y el entrenamiento de los observadores. También este tipo de escala solo evaluó las conductas en cuanto a su frecuencia, no conociendo si disminuye la intensidad o magnitud. Además, los esquemas de evaluación y codificación de las conductas antisociales no pueden haber sido lo suficientemente sensibles como para captar todos los cambios que se presentaron en los adolescentes.

Se necesita de más investigación para determinar que componentes de la intervención llevan a la disminución de las conductas antisociales y que elementos de esta se pueden ajustar con el fin de promover de forma consistente estos cambios. También es difícil determinar si el uso de todos los componentes de la intervención combinada llevaría a la disminución de estas conductas como al aumento de los índices de felicidad y optimismo. Es posible que los cambios en las conductas antisociales se deban a alguna otra variable que no midió el estudio, por lo que futuras investigaciones deben considerar la presencia de factores inespecíficos existentes en el proceso de evaluación y tratamiento como la deseabilidad social, ya que cada diada tenía conocimiento de estar participando de una investigación.

Los estudios futuros deben extender la duración de la fase de seguimiento para examinar si la disminución de las conductas antisociales y el aumento de la felicidad y optimismo son permanentes en el tiempo.

\section{CONCLUSIONES}

El presente estudio responde a la necesidad de contar con nuevas estrategias psicológicas específicas y evaluadas que ayuden a disminuir las conductas antisociales en adolescentes, debido a que este trastorno en la actualidad es altamente frecuente en esta población y constituyen un problema de relevancia para los profesionales de la salud. Cobra relevancia si se considera la necesidad de una intervención temprana, eficaz y efectiva, ya que, esta conducta tiende a ser estable en el tiempo y se constituye en un predictor de problemas y alteraciones en la etapa adulta.

La intervención fomento el fortalecimiento de emociones positivas como la felicidad y de fortalezas como el optimismo, lo que se visualiza necesario para este grupo etario, lo cual se realiza en un corto plazo y con permanencia en el tiempo.

Finalmente, este estudio constituye un acercamiento en Chile al ámbito de las conductas antisociales en adolescentes desde la psicología positiva, la cual aporta una herramienta útil y concreta que en corto tiempo y a bajo costo, da respuesta a este tipo de problema.

\section{Declaración de conflicto de intereses}

Los autores declaran no tener ningún conflicto de interés potencial con respecto a la investigación, la autoría, y/o publicación del presente artículo. 


\section{REFERENCIAS BIBLIOGRÁFICAS}

Andreu, J.M., Peña, M.E., \& Larroy, C. (2010). Conducta antisocial, impulsividad y creencias justificativas: análisis de sus interrelaciones con la agresión proactiva y reactiva en adolescentes. Behavioral Psychology, 18, 57-72.

Blatný, M., Hrdli ka, M., Jelínek, M., \& Sobotková, V. (2012). Antisocial behavior in adolescence: Typology and relation to family context. Journal of Early Adolescence, 33(8), 1091-1115. https://doi.org/10.1177/027243 1612445377

Bohlmeijer, E.T., Bolier, L., Lamers, S.M.A. \& Westerhof, G.J. (2017). Intervenciones clínicas positivas: ¿por qué son importantes y cómo funcionan? Papeles del Psicólogo, 38(1), 34-41. https://doi.org/10.23923/pap.psicol2017.2819

Carrea, G. \& Mandil, J. (2011). Aportes de la psicología positiva a la terapia cognitiva infanto juvenil. PSIENCIA, 3(1), 40-56.

Chavarín-García, M.C. \& Gálvez-Hernández, C. (2018). Conducta antisocial adolescente y dinámica familiar. Análisis conceptual. Psicología Iberoamericana, 26(1), 11-21.

Chico, E. (2002). Optimismo disposicional como predictor de estrategias de afrontamiento. Psicothema, 14(3), 544-550.

Díaz García, N. \& de la Villa Moral-Jiménez, M. (2018). Consumo de alcohol, conducta antisocial e impulsividad en adolescentes españoles. Acta Colombiana de Psicología, 21(2), 110-120. http://www.dx.doi.org/10.14718/ACP.2018.21.2.6

Diener, E., \& Seligman, M.E.P. (2002). Very Happy People. Psychological Science, 13(1), 81-84. https://doi.org/10.1111/1467-9280.00415

Donaldson, S. I., Dollwet, M., y Rao, M. (2015). Happiness, excellence, and optimal human functioning revisited: Examining the peer-reviewed literature linked to positive psychology. The Journal of Positive Psychology, 10,185-195.

Estévez, E. \& Emler, N.P. (2011). Assesing the links among adolescent and youth offending, antisocial behaviour, victimization, drug use and gender. International Journal of Clinical and Health Psychology, 11(2), 269-289.

Fondo de las Naciones Unidas para la Infancia (2011). Estado Mundial de la Infancia 2011: La Adolescencia, Una época de oportunidades: Fondo de las Naciones Unidas para la Infancia.

Forehand, R. \& Mc Mahon, R. (1981). A clinician's guide to parent training: Guilford Press.

Fredrickson, B.L. (2001). The role of positive emotion in positive psychology: The broaden and build theory of positive emotion. American Psychologist, 56, 218-226.

Garaigordobil, M. (2017). Conducta antisocial: conexión con bullying/cyberbullying y estrategias de resolución de conflictos. Psychosocial Intervention, 26, 4754. https://doi.org/10.1016/j.psi.2015.12.002

Garaigordobil, M. \& Maganto, C. (2016). Conducta antisocial en adolescentes y jóvenes: prevalencia en el País Vasco y diferencias en función de variables socio-demográficas. Acción psicológica, 13(2), 57-68. https://doi.org/10.5944/ap.13.2.17826

Gázquez, J. J., Pérez-Fuentes, M. C., Molero, M. M., Barragán, A. B., Martos, A., \& Sánchez, C. (2016). Drug use in adolescents in relation to social support and reactive and proactive aggressive behavior. Psicothema, 28(3), 318- 322. http://dx.doi.org/10.7334/psicothema2015.327

Giménez, M., Vázquez, C. \& Hervás, G. (2010). El análisis de las fortalezas psicológicas en la adolescencia: Más allá de los modelos de vulnerabilidad. Psychology, Society, \& Education, 2(2), 97-116.

Hasking, P.A., Scheier, L.M. \& Abdallah, A.B. (2011). The three latent antisocial classes of adolescent delinquency and the risk factors for membership in each class. Aggressive behaviour, 37, 19-35. 
Kahneman, D., Krueger, A. B., Schkade, D., Schwarz, N., \& Stone, A. A. (2006). Would you be happier if you were richer? A focusing illusion. Science 312(5782), 1908-1910. https://doi.org/10.1126/science.1129688

Kazdin, A.E. (2001). Métodos de investigación en psicología clínica: Pearson Educación.

López, S., \& Rodríguez-Arias, J. L. (2010). Factores de riesgo y de protección en el consumo de drogas en adolescentes y diferencias según edad y sexo. Psicothema, 22(4), 568-573.

Lyubomirsky, S., King, L. A., y Diener, E. (2005). The benefits of frequent positive affect: Does happiness lead to success? Psychological Bulletin, 131, 803-855

Lyubomirsky, S., \& Lepper, H.S. (1999). A measure of subjective happiness: Preliminary reliability and construct validation. Social Indicators Research, 46, 137-155.

Martínez, V. (2010). Informe Final: Caracterización del perfil de niños, niñas y adolescentes, atendidos por los centros residenciales de SENAME: Fondo de las Naciones Unidas para la Infancia.

Morán, C. Carmona, J., \& Fínez, M. (2016). Tipos de personalidad, agresión y conducta antisocial en adolescentes. Psychology, Society, \& Education, 8(1), 65-80. https://doi.org/10.25115/psye.v8i1.548

Nasaescu, E., Zych, I., Ortega-Ruiz, R., Farrington, D., \& Llorent, V. (2020). Longitudinal patterns of antisocial behaviors in early adolescence: a latent class and latent transition analysis. The European Journal of Psychology Applied to Legal Context, 12(2), 85-92. https://dx.doi.org/10.5093/ejpalc2020a10

Otero, J.M., Luengo, A., Romero, E., Gómez, J.A. \& Castro, C. (1998). Psicología de personalidad. Manual de prácticas: Ariel Practicum.

Piotrowska, P., Stride, C., Croft, S., \& Rowe, R. (2015). Socioeconomic status and antisocial behaviour among children and adolescents: A systematic review and meta-analysis. Clinical Psychology Review, 35, 47-55. https://doi.org/10.1016/j.cpr.2014.11.003

Rashid, T. (2015). Positive psychotherapy: A strength-based approach. Journal of Positive Psychology, 10, 25-40.

Ribas, M., del Prado, N., Claramunt, J., Civit, M., Canalias, O., \& Santaolalla, A., (2015). Adolescentes multiproblemáticos: consumo de tóxicos y trastorno mental en jóvenes que delinquen. Actas Españolas de Psiquiatría, 43(6), 197-204

Rodríguez, F. J., Ovejero, A., Bringas, C., \& Moral, M. V. (2016). Afrontamiento de conflictos en la socialización adolescente. Propuesta de un modelo. Psicología desde el Caribe, 33(1), 1-13. DOI: http://dx.doi.org/10.14482/psdc.33.1.8083

Rodríguez, F. J., Rodríguez-Franco, I., López-Cepero, J., \& Bringas, C. (2012). Juvenile delinquency and Young ofender: bibliographical and bibliometric review of two perspectives of study. The European Journal of Psychology Applied to Legal Contex, 2(2), 117-143.

Salanova, M y Llorens, S. (2016). Hacia una psicóloga positiva aplicada. Papeles del psicólogo, 37(3), 161-164.

Scheier, M.F., Carver, C.S., \& Bridges M.W. (1994). Distinguishing optimism from neuroticism (and trait anxiety, self-mastery, and self-esteem): A re-evaluation of the Life Orientation Test. Journal of Personality and Social Psychology, 67, 1063-1078.

Seligman, M. E. P. (2008). Positive Health. Applied Psychology: An International review, 57, 3-18.

Seligman, M. E. P., Rashid, T., y Parks, A. C. (2006). Positive psychotherapy. American Psychologist, 61(8), 774.

Seligman, M. E., Steen, T. A., Park, N., y Peterson, C. (2005). Positive psychology progress Empirical validation of interventions. American Psychologist, 60, 410-421.

Servicio Nacional de Menores. (2010). Bases técnicas, líneas de acción centros residenciales modalidad residencias de protección para mayores: Servicio Nacional de Menores. 
Servicio Nacional de Menores (2019). Orientación técnica: residencia familiar de administración directa para adolescentes: Servicio Nacional de Menores.

Sheldon, K. M., Kasser, T., Smith, K., \& Share, T. (2002). Personal goals and psychological growth: testing an intervention to enhance goal attainment and personality integration. Journal of personality, 70(1), 5-31. https://doi.org/10.1111/1467-6494.00176

Toribio, L., \& González-Arratia, N., Van Barneveld, H. \& Gil, M. (2018). Salud mental positiva en adolescentes mexicanos: diferencias por sexo. Revista Costarricense de Psicología, 37(2),131-143

Vera-Villarroel, P., Celis-Atenas, K \& Córdova-Rubio, N. (2011). Evaluación de la Felicidad: Análisis psicométrico de la escala de felicidad subjetiva en población chilena. Terapia Psicológica, 29(1), 127-133.

Vera-Villarroel, P., Córdova-Rubio, N., \& Celis-Atenas, K. (2009). Evaluación del optimismo: un análisis preliminar del Life Orientation Test versión revisada (LOT-R) en población chilena. Universitas Psychologica, 8, 61-68. 